\title{
THE INFLUENCE OF PHYSIOTHERAPY ON GAIT AFTER SPINAL SURGERY
}

\author{
Tsvetelina Bizheva \\ National Sports Academy "V. Levski”
}

\begin{abstract}
Summary:
Purpose. The purpose of the study is to determine the influence of specialized physiotherapy program on gait in patients after lumbar spinal surgery.

Methodology The contingent includes - 80 patients, of which 38 males and 42 females. The assessment was made on the first day after surgery, on the day of discharge and one month after surgery. The gait was examined with a Functional gait assessment (FGA) test and 6 meters walking test. All patients underwent daily exercises including proprioceptive neuromuscular facilitation (PNF) during hospital stay and performed a home exercise program for one month after discharge.

Results There were significant improvements in the first month in all functional aspects of the gait.

Discussion The specialized early physical therapy including PNF and home exercise program leads to improved gait and walking speed.

Conclusions The study showed that the use of physiotherapy during hospital stay and one month after surgery positively influenced the functionality and speed of walking in patients after spinal surgery.
\end{abstract}

Keywords: spinal surgery, gait, physiotherapy

\section{Introduction:}

Herniated lumbar disc is a displacement of disc material beyond the intervertebral disc space. The highest prevalence is among people aged 30 to 50 years, with a male to female ratio of 2:1 (Jordan et al, 2011). Surgical intervention is the most common method used to treat spinal disc herniation. When surgery is performed with adequate consideration for the resulting adaptation syndromes its success rates are from 80 to $90 \%$ (Ju et al., 2012). For better surgical outcomes and avoidance of unpleasant complications, the most important points are appropriate patient selection and effective neural decompression constitute (Omidi-Kashani et al, 2016). Because the surgical removal of the disc and nerve decompression does not resolve the whole pathological process of the lumbar disorder, post-operative exercise treatment programs for rehabilitation are important (Ju et al, 2012).

There are numbers of rehabilitation programs for recovering after spinal surgery, but almost all of them are targeting the improvement of the movements, increasing the strength and the endurance of the muscles and improving the performance of everyday task (Yoo, 2015) and (Sokunbi, Kachalla 2015). There is very little information about what the actions must be in order to improve the gait in patients after lumbar spinal surgery.

The current study is researching the exercises that help to improve the gait. Walking exercises are a good choice for such, because walking is well tolerated by most patients regardless of fitness level and back condition.

Aim and objectives of the study. The purpose of the study is to evaluate the effect of application of specialized physiotherapy program on gait in patients after lumbar spinal surgery. The contingent includes -80 patients, of which 38 males and $42 \mathrm{fe}$ males, voluntarily attended, treated in Department of neurosurgery of the University Hospital Sofiamed-Sofiq. The mean age of patients is 61 years.

Methods: The assessment was made on the first day after surgery, on the day of discharge and one month after surgery. The gait was examined with a Functional gait assessment (FGA) test and 6 meters walking test. All patients performed daily physiotherapy program for 30 minutes with mild to moderate intensity until the day of discharge. PNF for gait training was used during the hospital stay. After discharge all patients did a walking program at home for one month.

The main goal was to gradually extend activities of daily living from personal care to housekeeping tasks in the short term and return to work in the long term. At the beginning the treatment was focused on the ability and possibility to execute personal care activities and perform transfers in bed. Next step was gradually increasing the load of the exercises, using lumbar extension muscle-strengthening pro- 
gram, exercise in walking, climbing stairs and stepping over obstacle. The functional gait assessment test was used as a part of the rehabilitation program.

Statistical analysis was performed using SPSS19. 00 for Windows. Paired sample t-test was used to determine the effect of the intervention on Gait and speed.

Results: There were significant improvements in the first month in all functional aspects of the gait. The highest improvement was seen in indicators: step over obstacle with 0.97 points, gait end pivot turns with 0.91 point and change in gait speed with 0.90 points. The rest of the indicators improve with 0,72 to 0.88 points. (fig. 1 ) The overall point for FGA at the beginning were 14.25. After one month there was a significant improvement to 21.65 (fig.2). The 6 meters walking test shows significant improvements on the day of discharge and one month after surgery (fig.3).

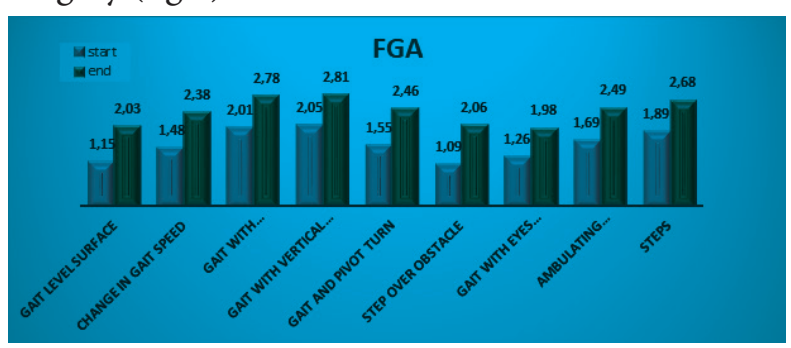

Fig.1 Changes in the subscales of FGA before and after applied physical therapy

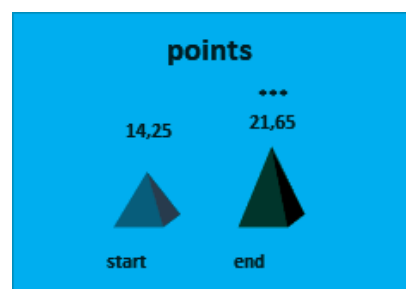

Fig.2 Changes in the FGA overall points

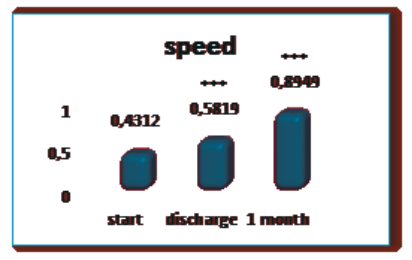

Fig.3 Changes in speed at the beginning, on before and after applied physical therapy the day of discharge and one month after surgery

\section{Discussion:}

According to Oosterhuis end Tulder, patients who undergo lumbar disc surgery for herniated discs, are advocated two different postoperative management strategies: a watchful waiting policy, or referral for rehabilitation immediately after discharge from the hospital. (Oosterhuis et all, 2013). We started our physiotherapy program at the very first day after surgery. At the beginning the intensity was low and the goals were painless turning in bed, early verticalization and performing a few steps. We believe that the immediate engage with the program was reason for the observed significant increase in the walking speed in all of our patients at the day of discharge. The starting and ending values are shown in fig. 3. Our results are similar to those of Erdogmus, which proof that physiotherapy-based rehabilitation starting 1 week after lumbar disc surgery is more effective in the short-term (Erdogmus et al, 2007).

Hylton suggest that one of the primary objectives of the postural control system when walking is the head control, and that subjects adapt their stepping pattern on irregular surfaces to ensure that the head remains stable (Hylton et al, 2003). Based on that, we consider that the position and the movements of the head have special part during the rehabilitation. The FGA test itself include walking while looking left and right, up and down, walking over obstacle, walking with closed eyes also walking with reversal of direction and walking backwards. In our research we used the FGA test not only for assessment, but as a daily exercise. On that base we report considerable rising in all of its components, as shown in fig. 1. During the study we also noticed that all patients after back surgery are walking with a bowed head due to insecurity and fear of falling. Applying the FGA test as a daily exercise trains the patients to look at all directions, while building confidence and decreasing the fear of falling.

Studies on the effects of treadmill walking exercises for treating post-surgical lumbar disc herniation are not many, but in some of them is mentioned that the therapeutic benefit of treadmill exercises might be similar to the benefit of core-stability exercises. The study of Sokunbi showed that a combination of core-stability exercises, and treadmill walking exercises produced improvement in terms of pain reduction, increased spinal mobility, and improved general health of the patient following lumbar disc herniation surgery (Sokunbi, Kachalla,2015). In our study we combined lumbar extension muscle-strengthening program along with a 
walking program with the FGA. An improvement of the mentioned above indicators, for a period of one month after spinal surgery, which is also supported from more studies, as those of Choi, who concluded that the early lumbar extension muscle-strengthening program has positive effects in patients after herniated lumbar disc surgery (Choi et al, 2005). The results of previous research together with this study's findings suggest that the performance of lumbar extension muscle-strengthening combined with gait training may significantly improve the walking sped and the possibilities for walking as general for patients after lumbar disc herniation surgery.

\section{Conclusions}

The study showed that the application of physiotherapy program during hospital stay and one month after surgery positively influenced the functionality and speed of walking in patients after spinal surgery.

The survey we made is only one aspect of broad field for research. There must be more surveys targeting the changes in the gait after spinal surgery. The possibilities of the impact of the physiotherapy has to be considered, too.

Therefore, this program must be introduced as a rehabilitation program after lumbar disc herniation surgery.

\section{References:}

Choi G, Raiturker PP, Kim MJ, et al. (2005). The effect of early isolated lumbar extension exercise program for patients with herniated disc undergoing lumbar discectomy. Neurosurgery, 2005, 57: 764-772.

Erdogmus C, Resch K, Sabitzer R, Müller H, Nuhr M, Schöggl A, et al. Physiotherapy-based rehabilitation following disc herniation operation: results of a randomized clinical trial. Spine. 2007; 32:2041-2049.
Hylton B. Menz, Stephen R. Lord, Richard C. Fitzpatrick. (2003). Acceleration patterns of the head and pelvis when walking on level and irregular surfaces Gait end posture August 2003Volume 18, Issue 1, Pages 35-46 Jordan, J., Konstantinou, K., and O’Dowd, John. (2011). Herniated lumbar disc, Clinical Evidence Musculoskeletal disorders BMJ Publishing Group Ltd 2011.06:1118

Ju S, Park G, Kim E. (2012). Effects of an exercise treatment program on lumbar extensor muscle strength and pain of rehabilitation patients recovering from lumbar disc herniation surgery. J Phys Ther Sci. 2012; 24:515518.

Omidi-Kashani, F., Hejrati, H., and Ariamanesh, S. (2016) Ten Important Tips in Treating a Patient with Lumbar Disc Herniation, Asian Spine J. 2016 Oct; 10(5): 955-963

Oosterhuis T, van Tulder M, Peul W, Bosmans J, Vleggeert-Lankamp C, Smakman L, et al. (2013). Effectiveness and cost-effectiveness of rehabilitation after lumbar disc surgery (REALISE): design of a randomised controlled trial. BMC Musculoskelet Disord. 2013; 14:124

Sokunbi, G., Kachalla, G., Effects of Acupuncture, Core-stability Exercises, and Treadmill Walking Exercises in Treating a Patient with Postsurgical Lumbar Disc Herniation: A Clinical Case Report, J Acupunct Meridian Stud 2015;8(1):48e52

Sabitzer R, Müller H, Nuhr M, Schöggl A, et al. (2007). Physiotherapy-based rehabilitation following disc herniation operation: results of a randomized clinical trial. Spine. 2007; 32:2041-2049.

Yoo, W., (2015). Comparison of the isolated contraction ratios of the hip extensors and erector spinae muscles of the lumbar region and thoracic muscles during different back extension exercises J. Phys. T 316 her. Sci. Vol. 27, No. 2, 2015,

Contact information with the corresponding author: Bizheva Tsvetelina Stanimirova, assistant

National Sports Academy "V. Levski"

Department of Kinesitherapy and Rehabilitation

Bulgaria, Sofia

str. «Gurgulqt» № 1 ,

1000 Sofiq,

Telephone number: +359886743213

e-mail: tsvetelina bijeva@abv.bg 